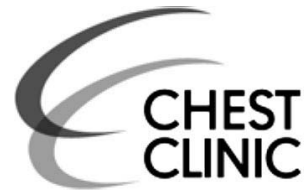

Department of Internal Medicine, Jikei University School of Medicine, Minatoku, Tokyo, Japan

\section{Correspondence to} Dr Hiromichi Hara, Division of Respiratory Diseases, Department of Internal Medicine, Jikei University School of Medicine, 3-19-18 Nishishimbashi, Minatoku, Tokyo 105-8471, Japan;

hirohara@jikei.ac.jp

Received 19 October 2016 Revised 11 November 2016 Accepted 12 November 2016 Published Online First 2 December 2016

\title{
Macroscopic inflammatory tracheal and endobronchial nodules in Sjögren's syndrome
}

\author{
Hiromichi Hara, Kazuyoshi Kuwano, Shunsuke Minagawa, Takanori Numata, \\ Jun Araya, Katsutoshi Nakayama
}

A 38-year-old woman with primary Sjögren's syndrome (SS) presented with a 1 month history of cough and chest pain. She had been treated with prednisolone for organising pneumonia and had been on prednisolone $(5 \mathrm{mg} /$ day $)$. Flexible bronchoscopy revealed macroscopic multiple nodules mainly in trachea and in both main bronchus (figure 1A). Three-dimensional CT (3D-CT) also demonstrated multiple nodules in the same place (figure 1B). Endobronchial biopsy showed polyclonal lymphocyte infiltration in the bronchial submucosa and epithelium in part (figure $1 \mathrm{C}, \mathrm{H} \& \mathrm{E}$ : upper, CD3: middle, CD20: lower). There were few IgG4-positive plasma cells in the lesion. Amyloid deposition was not detected by direct fast scarlet staining. Abnormal proliferations of squamous epithelium were not observed, and human papilloma virus DNA was not detected in the bronchial lavage fluid. These findings indicated that the inflammatory nodules were associated with SS. Selective cyclo-oxygenase- 2 inhibitor, celecoxib, was only added to her medication, and her symptoms disappeared within several weeks. Without addition of any other treatment, multiple nodules dramatically regressed, which was demonstrated by repeated bronchoscopic examination performed 5 months later (figure 1A).

SS is a common autoimmune disease, characterised by lymphocytic infiltration of exocrine glands. SS also affects a variety of extraglandular organs. Infiltration of $\mathrm{T}$ and $\mathrm{B}$ cells into affected tissues is a characteristic pathology of SS although it is not disease specific. We concluded that the inflammatory nodules in the trachea and bronchus were due to SS by clearly excluding other diseases that form endobronchial nodules including malignant lymphoma, IgG4-related diseases, amyloidosis and papilloma.

Airway is commonly affected in SS regardless of the presence of clinical symptoms. ${ }^{1}{ }^{2}$ Among airway diseases in SS, peripheral airway diseases including bronchiolitis and bronchiectasis are frequently observed; however, central airway lesions in SS, especially with macroscopic nodules, are rare. ${ }^{3}$ Without bronchoscopy or 3D-CT, central airway lesions in SS are usually overlooked and hence central airway may be more frequently involved in SS than previously reported. Specific mechanisms of formation of macroscopic nodules in central airway in SS remain to be understood.

Triggers of formation and resolution of the inflammatory nodules were not determined in the present case. Viral infection is considered as a trigger of increased activity of SS and hence we speculate that transient viral infection might contribute to the formation of the nodules through modulating SS activity. In autoimmune diseases, disease activities fluctuate and diseased lesions sometimes spontaneously regress. Even lymphoma associated with SS (lymphocytes are more proliferative than the present case) resolve spontaneously in some cases. Therefore, spontaneous resolution of inflammatory nodules associated with SS is quite possible.

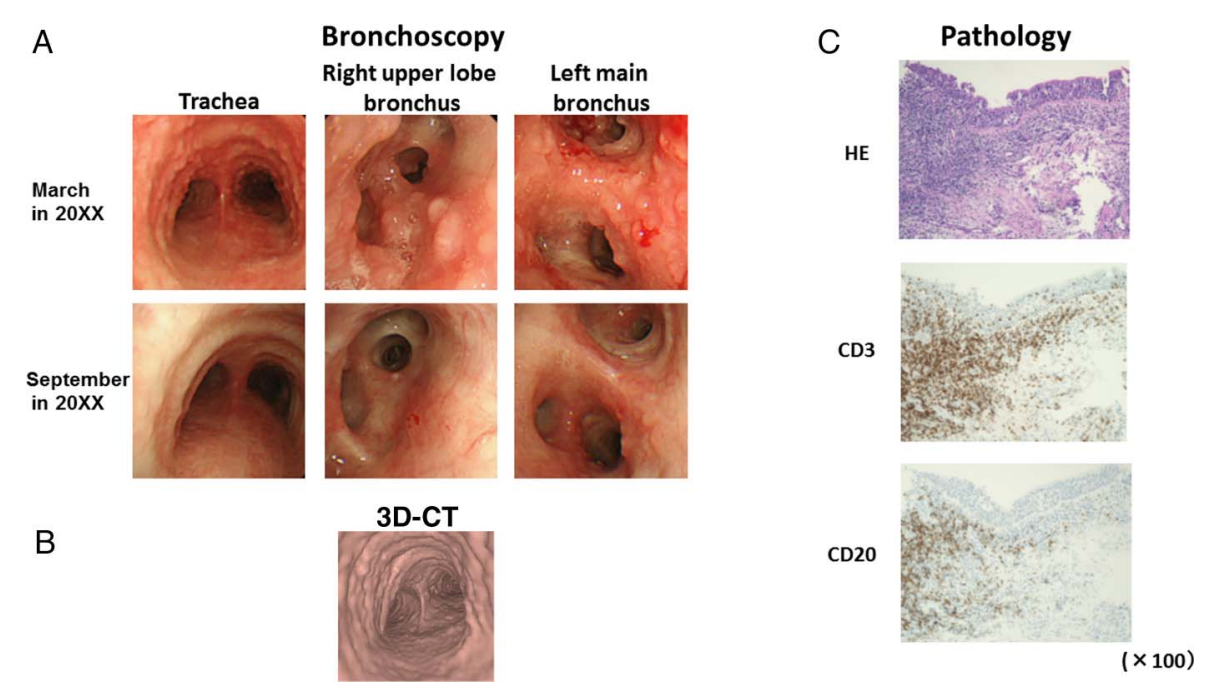

To cite: Hara H, Kuwano $\mathrm{K}$ Minagawa S, et al. Thorax 2017;72:864-865.

Figure 1 (A) Bronchoscopic findings (trachea, right upper lobe, left main bronchus). (B) Three-dimensional CT (3D-CT) of trachea. (C) Pathology of multiple nodules (endobronchial biopsy). 
In conclusion, we demonstrated macroscopic inflammatory tracheal and endobronchial nodules in SS determined by bronchoscopy and 3D-CT.

Contributors All authors wrote and revised the manuscript.

Competing interests None declared.

Patient consent Obtained.

Provenance and peer review Not commissioned; externally peer reviewed.

\section{REFERENCES}

1 Kreider M, Highland K. Pulmonary involvement in Sjögren syndrome. Semin Respir Crit Care Med 2014;35:255-64.

2 Papiris SA, Saetta M, Turato G, et al. CD4-positive T-lymphocytes infiltrate the bronchial mucosa of patients with Sjögren's syndrome. Am I Respir Crit Care Med 1997;156(2 Pt 1):637-41.

3 Miyazaki N, Kido M, Kajiki A, et al. Endobronchial pseudolymphoma associated with Sjögren's syndrome. Report of a case. Endoscopy 1989;21:99-101. 